ISSN 1980-5098 @ (ब\& DOI: http://dx.doi.org/10.5902/1980509831580

\title{
MODELO 3-PG NA PREVISÃO DO POTENCIAL PRODUTIVO DE ÁREAS PARA PLANTIOS COMERCIAIS DE Eucalyptus spp.
}

\author{
3-PG MODEL IN PREDICTIING THE PRODUCTIVE POTENTIAL AREAS FOR COMMERCIAL \\ STANDS OF Eucalyptus spp.
}

Carlos Magno Moreira de Oliveira ${ }^{1}$ Rafael Coll Delgado ${ }^{2}$ Emanuel José Gomes de Araújo $^{3}$ André Quintão de Almeida ${ }^{4}$ Thais Cristian Rosa ${ }^{5}$ José Francisco de Oliveira Júnior ${ }^{6}$

\section{RESUMO}

A predição da produção florestal requer conhecimento de variáveis dendrométricas e edafoclimáticas. Esse trabalho teve por objetivo ajustar e avaliar a precisão do modelo 3-PG em estimar as variáveis altura, diâmetro a altura do peito (DAP), volume e incremento médio anual (IMA) em plantio comercial de Eucalyptus spp. no norte de Minas Gerais, utilizando dados meteorológicos provenientes de estações automáticas de superfície e de sensoriamento remoto. Para validação do modelo foram estimados os dados de altura, DAP, volume e IMA pelo 3-PG e comparados com dados de inventários florestais, totalizando 335 medições, em 10 talhões, realizadas no período de 2005 a 2011. Foram aplicados os métodos estatísticos correlação linear (Pearson - r), coeficiente de determinação $\left(\mathrm{R}^{2}\right)$, erro padrão da estimativa $\left(\mathrm{S}_{\mathrm{yx}}\right)$, erro padrão da estimativa em porcentagem $\left(\mathrm{S}_{\mathrm{yx}} \%\right)$ e eficiente do modelo $(\mathrm{EF})$. Observou-se que o modelo tende a superestimar as variáveis dendrométricas quando se utiliza a parametrização proposta para região norte de Minas Gerais. Após o ajuste dos parâmetros relativos à idade de fechamento do dossel (fullCanAge), a relação entre stem e DAP (nS) e parametrização das equações alométricas relativas à altura total e ao volume de madeira, incorporado no 3-PG, o modelo obteve resultados satisfatórios com valores de $\mathrm{R}^{2}=0,94$ e EF $=0,87$ para DAP, altura e volume e $\mathrm{R}^{2}=0,85$ e $\mathrm{EF}=0,58$ para o IMA, assim como apresentou distribuição dos dados estimados próximo aos valores médios dos dados observados. O 3-PG mostrou eficiência na estimativa do DAP, altura, volume e IMA, tornando-se ferramenta potencial para avaliação de áreas propícias para expansão de novos plantios.

Palavras-chave: produção florestal; modelagem baseada em processos; modelo ecofisiológico.

\section{ABSTRACT}

The prediction of a forest production requires knowledge of the dendrometric and edaphoclimatic variables. This study aimed to adjust and evaluate the accuracy of the 3-PG model in estimating the variables height, diameter at breast height (DBH), volume and mean annual increment (MAI) in commercial plantations of Eucalyptus spp. in northern of Minas Gerais state, using meteorological data from automatic surface stations and remote sensing. To validate the model, data of height, DBH, volume and MAI by 3-PG were

1 Engenheiro Florestal, MSc., Professor do Instituto Federal do Norte de Minas Gerais, Campus Arinos, Rodovia MG 202, Km 407, Arinos, Caixa Postal 05, CEP 38680-000, Arinos (MG), Brasil. carlos.moreira@ifnmg.edu.br

2 Meteorologista, Dr., Professor Adjunto do Departamento de Ciência Ambientais, Universidade Federal Rural do Rio de Janeiro, BR 465, Km 07, CEP 23890-000, Seropédica (RJ), Brasil. rafaelcolldelgado32@gmail.com / junior_inpe@hotmail.com

3 Engenheiro Florestal, Dr., Professor Adjunto do Departamento de Silvicultura, Universidade Federal Rural do Rio de Janeiro, BR 465, Km 07, CEP 23890-000, Seropédica (RJ), Brasil. emanuelaraujo@ufrrj.br

4 Engenheiro Florestal, Dr., Professor Adjunto do Departamento de Engenharia Agrícola, Universidade Federal de Sergipe, Av. Marechal Rondo, S/N, Jardim Rosa Elze, CEP 49100-000, Sergipe (SE), Brasil. andreqa@gmail.com

55 Engenheira Florestal, Vallourec Florestal Ltda. Rua Honduras, 78 - Bairro Léo Batista - 35790-000 - Curvelo, MG, Brasil. E-mail: thais.rosa@vallourec.com

6 Meteorologista, Dr., Professor Adjunto do Instituto de Ciências Atmosféricas (ICAT), Universidade Federal de Alagoas, Av. Lourival Melo Mota, s/n, Tabuleiro dos Martins, CEP 57072-900, Maceió (AL), Brasil. jose.junior@ icat.ufal.br

Recebido para publicação em 31/10/2015 e aceito em 17/11/2016

Ci. Fl., v. 28, n. 1, jan.- mar., 2018 
estimated and compared with forest inventory data, totaling 335 measurements in 10 plots in the period 2005 to 2011. Linear statistical correlation methods (Pearson - r), the coefficient of determination $\left(\mathrm{R}^{2}\right)$, the standard error of estimate $\left(\mathrm{S}_{\mathrm{yx}}\right)$, the standard error of the estimate in percentage $\left(\mathrm{S}_{\mathrm{yx}} \%\right)$ and the efficient model (EF) were applied. It was observed that the model tends to overestimate the dendrometric variables when using the parameterization proposal for northern region of Minas Gerais state. After adjusting the parameters for the age of canopy closure (fullCanAge), the relationship between stem and DBH (nS) and parameterization of allometric equations for the total height and volume of wood incorporated in the 3-PG, obtained satisfactory results with values of $\mathrm{R}^{2}=0.94$ and $\mathrm{EF}=0.87$ for $\mathrm{DBH}$, height and volume and $\mathrm{R}^{2}=$ 0.85 and $\mathrm{EF}=0.58$ for the MAI, as presented distribution of estimated data close to the average values of the observed data. The 3-PG was shown to be effective in estimating the DBH, height, volume and MAI become a potential tool for evaluating suitable areas for the expansion of new crops.

Keywords: forest yield; process-based models; ecophysiological model.

\section{INTRODUÇÃO}

O gênero Eucalyptus tem sido muito utilizado no Brasil para atender à demanda de madeira para serraria, celulose, energia, dentre outros usos. Com isso, é cada vez mais comum o uso de ferramentas que viabilizem o entendimento das relações entre variáveis fisiológicas, edáficas e climáticas que regem o crescimento da árvore, principalmente em áreas extensas em que diversos fatores ambientais influenciam no desenvolvimento do plantio (ALMEIDA et al., 2004).

Modelos de crescimento e produção são ferramentas utilizadas para predizer o crescimento de plantios florestais comerciais. Porém, os modelos biométricos, tradicionalmente aplicados, possuem limitações para estimativa da produção em locais em que não há plantios, pois necessitam de medições temporais de variáveis dendrométricas. Outra característica dos modelos tradicionais é a baixa sensibilidade às variações climáticas anuais e se houver práticas de manejo diferentes durante as rotações do plantio, as relações empíricas são alteradas (STAPE; RYAN; BINKLEY, 2004).

Uma alternativa aos modelos tradicionais são os modelos baseados em processos ou modelos mecanísticos que calculam a fotossíntese, em princípio, pela estimativa da quantidade de Radiação Fotossinteticamente Ativa (RFA) absorvida pelo dossel e a capacidade deste em fixar carbono com base no parâmetro de eficiência do uso da luz, que por sua vez é regulado por fatores ambientais que afetam a condutância estomática ou atividade fotossintética (LANDSBERG; WARING, 1997). Dessa forma, os Modelos Baseados em Processos (MBP) podem ser definidos como um procedimento no qual o comportamento do sistema é considerado como função de um conjunto de componentes e de suas interações com o ambiente, por meio de processos físicos e mecânicos que ocorrem ao longo do tempo (MÄKELÄ et al., 2000).

A concepção sobre a aplicação dos MBPs tem mudado, o que tem contribuído para difusão desses modelos entre as empresas florestais. Contudo, ainda se tem a ideia de que os modelos empíricos fornecem resultados melhores quando comparados aos modelos mecanísticos (MÄKELÄ et al., 2000; TICKLE et al., 2001), isso porque estes têm capacidade de predição com maior objetividade em áreas amostradas, enquanto os processuais são mais generalistas, sendo potencialmente empregados em locais ainda sem informações quanto ao desenvolvimento de essências florestais de rápido crescimento (MAESTRI, 2003).

O entendimento sobre a atividade das florestas está sendo cada vez mais compreendido por meio de modelos baseados em processos fisiológicos que são testados em locais em que o gás carbônico $(\mathrm{CO} 2)$ e a água $(\mathrm{H} 2 \mathrm{O})$ estão sendo monitorados. Existe uma variedade de modelos que vem relacionando estimativas da Produtividade Primária Bruta (PPB), respiração autotrófica e o residual da Produtividade Primária Liquida (PPL); dentre eles estão o FOREST-BGC (RUNNING; COUGHLAN, 1988), BIOMASS (MCMURTRIE; ROOK; KELLIHER, 1990), TREGRO (WEINSTEIN; BELOIN; YANAI, 1991) e o 3-PG (Physiological Principles Predicting Growth) desenvolvido por Landsberg e Waring (1997).

Um dos grandes impedimentos para difusão e utilização dos modelos baseados em processos está na quantidade de parâmetros necessários para executar o modelo, sendo que alguns desses parâmetros são de difícil mensuração, como alocação de carbono acima e abaixo do solo, (ALMEIDA et al., 2004, CAMPION; ESPREY; SCHOLES, 2005), o que reduz a confiabilidade das estimativas, comparado aos 
modelos empíricos (MÄKELÄ et al., 2000). Além disso, os modelos baseados em processos são vistos apenas como ferramentas aplicáveis ao entendimento do desenvolvimento do ecossistema e para estimativa de produção de biomassa, ao invés de estimar variáveis dendrométricas de interesse dentro do manejo florestal, como diâmetro médio e volume (TICKLE et al., 2001).

Para ser integrado no processo de gestão florestal junto aos modelos biométricos, o modelo 3-PG foi simplificado e desenvolvido em linguagem visual basic utilizando o Excel (SANDS; LANDSBERG, 2002) facilitando o uso e parametrização, o que permite determinar o potencial de produtividade de áreas sem plantios (STAPE; RYAN; BINKLEY, 2004) e fornecer orientações para realizar estimativas sobre o estoque de madeira frente às variações climáticas (ALMEIDA; LANDSBERG; SANDS, 2004).

O 3-PG tem sido empregado em plantios homogêneos em diferentes países para espécies do gênero Eucalyptus, Pinus e Douglas fir (TICKLE et al., 2001; SANDS; LANDSBERG, 2002; ALMEIDA; LANDSBERG; SANDS, 2004; STAPE; RYAN; BINKLEY, 2004; PAUL et al., 2007; NIGHTINGALE et. al., 2008; RODRÍGUEZ-SUÁREZ; IGLESIAS; DIAZ-FIERROS, 2010; STAPE et al., 2010; BORGES et al., 2012; WEI et al., 2014; LONDERO et al., 2015). A facilidade de manuseio do 3-PG tem possibilitado a simulação da produtividade potencial de novas áreas sob condições ambientais variadas (ALMEIDA et al., 2004; CAMPION; ESPREY; SCHOLES, 2005), a relação do crescimento com o balanço hídrico do solo (ALMEIDA; SANDS, 2015), efeitos da adubação e irrigação sobre a produção (STAPE et al.; 2004), a predição de propriedades do solo e índice de área foliar (COOPS; WARING; HILKER, 2012) e a estimativa da produtividade primária bruta (PPB) (COOPS et al., 2007; NOLÈ et al., 2013).

Nessa perspectiva, o objetivo deste trabalho foi ajustar o modelo 3-PG para povoamentos de Eucalyptus spp. e avaliar seu desempenho em estimar variáveis de interesse no manejo florestal, como diâmetro, altura e volume, utilizando dados climáticos temporais coletados em estações meteorológicas automáticas e obtidos por sensoriamento remoto, com baixo custo de obtenção, alta precisão e resolução espacial que abrange toda a área de estudo.

\section{MATERIAL E MÉTODOS}

\section{Área de estudo}

O trabalho foi desenvolvido no município de Bocaíuva, Minas Gerais, em plantios comerciais de Eucalyptus spp. A área é composta por dez talhões implantados no ano de 2004, com espaçamento de $3 \mathrm{x}$ 3 metros. O clima da região, segundo Köppen, é o "Aw" tropical de savana, inverno seco e verão chuvoso, com temperatura média anual de $24,1^{\circ} \mathrm{C}$ e chuva média anual entre $1.000 \mathrm{~mm}$ e $1.100 \mathrm{~mm}$ (DURÃES et al., 2014).

\section{Calibração do modelo 3-PG}

O modelo ecofisiológico 3-PGpjs 2.4 requer parâmetros relativos à fisiologia da espécie e parâmetros alométricos (SANDS; LANDSBERG, 2002). Inicialmente, foi utilizada a parametrização proposta por Borges et al. (2012) para Eucalyptus spp. no cerrado na porção norte (N) de Minas Gerais e avaliada a eficiência para estimativa das variáveis dendrométricas.

Posteriormente foi realizada a parametrização dos modelos alométricos relativos à altura $(\mathrm{m}) \mathrm{e}$ ao volume $\left(\mathrm{m}^{3} \cdot \mathrm{ha}^{-1}\right)$ e ajuste dos parâmetros fullCanAge e StemPower, sendo que os valores dos demais parâmetros sugeridos por Borges et al. (2012) foram mantidos. Por meio do diâmetro a altura do peito (DAP, cm), altura comercial e volume, coletados no período de 2001 a 2011 por meio de inventário florestal, totalizando 2030 observações, foram ajustadas equações para obtenção dos parâmetros constante de relação entre DAP e altura $(\mathrm{aH})$, potência de relacionamento entre DAP e altura $(\mathrm{nHB})$, constante de relacionamento entre volume e DAP $(\mathrm{aV})$ e potência de relacionamento entre volume e DAP (nVB). Segundo Landsberg e Waring (1997), os modelos alométricos incorporados no 3-PG relacionam a altura total da árvore e o diâmetro a altura do peito (DAP) (H), o volume total e o DAP (V), como seguem: 


$$
\begin{aligned}
& \mathrm{H}=a_{\mathrm{h}} \cdot \mathrm{DAP}^{\mathrm{n}_{\mathrm{h}}} \\
& \mathrm{V}=\mathrm{a}_{\mathrm{v}} \cdot \text { DAP }^{\mathrm{n}_{\mathrm{v}}}
\end{aligned}
$$

Em que: $H$ = variável altura total; $V=$ variável volume; $a_{h}$ e $a_{v}=$ coeficiente; $n_{h}$ e $n_{v}=$ potência que reflete a característica da espécie.

Com a parametrização dos modelos alométricos do 3-PG, as variáveis DAP, altura, volume e incremento médio anual (IMA), estimados pelo modelo 3-PG, foram comparadas com os dados obtidos pelo inventário florestal, a fim de ajustar os parâmetros fullCanAge que é relacionado à idade de fechamento do dossel, ou seja, quando se tem a máxima interceptação da RFA pelas folhas, e StemPower (nS) que relaciona a biomassa do stem (tronco + galhos + casca) e o DAP (RODRÍGUEZ-SUÁREZ; IGLESIAS; DIAZ-FIERROS, 2010).

\section{Dados de entrada do modelo}

Com relação aos dados de entrada, o modelo requer informações relativas à temperatura mínima $\left(t_{n}\right.$, $\left.{ }^{\circ} \mathrm{C}\right)$ e máxima do $\operatorname{ar}\left(\mathrm{t}_{\mathrm{x}},{ }^{\circ} \mathrm{C}\right)$, radiação solar global $\left(\mathrm{R}_{\mathrm{s}}, \mathrm{MJ} \mathrm{m}^{-2}\right.$. dia $\left.{ }^{-1}\right)$ e chuva $(\mathrm{mm})$ que podem ser inseridos no modelo na forma de médias históricas da região ou médias mensais atuais, sendo esta a escala dos dados empregados nesse trabalho. Dados relativos a $\mathrm{t}_{\mathrm{x}} \mathrm{e} \mathrm{t}_{\mathrm{n}}$ foram obtidos de Estações Meteorológicas Automáticas (EMA) do Instituto Nacional de Meteorologia (INMET), a chuva foi obtida do satélite tropical TRMM (Tropical Rainfall Measuring Mission) via produto 3B43, com resolução espacial de 20 km e chuva horária que foi convertida para médias mensais (OLIVEIRA JÚNIOR et al., 2014).

Os valores relativos a $\mathrm{R}_{\mathrm{s}}$ foram obtidos pelo método proposto pela FAO 56 (Food and Agriculture Organization of the United Nations) (ALLEN; JENSEN; BORNAN, 1989) que requerem informações de latitude do local e o número de horas de brilho solar por dia (n), sendo esta última obtida da base de dados do INMET:

$$
\mathrm{R}_{\mathrm{s}}=\left(\mathrm{a}_{\mathrm{s}}+\mathrm{b}_{\mathrm{s}} \frac{\mathrm{n}}{\mathrm{N}}\right) \cdot \mathrm{R}_{\mathrm{a}}
$$

Em que: $R_{s}=$ radiação solar $\left(\mathrm{MJ} \cdot \mathrm{m}^{-2} \cdot \operatorname{dia}^{-1}\right) ; \mathrm{a}_{\mathrm{s}}+\mathrm{b}_{\mathrm{s}}=$ fração da radiação no topo da atmosfera que chega ao planeta em dias claros ( $\mathrm{n}=\mathrm{N}) ; \mathrm{n}=$ duração de brilho solar (horas); $\mathrm{N}=$ possibilidade máxima de duração de horas de brilho solar (horas); $\mathrm{R}_{\mathrm{a}}=$ radiação solar no topo da atmosfera $\left(\mathrm{MJ} \cdot \mathrm{m}^{-2} \cdot \mathrm{dia}^{-1}\right.$ ).

A radiação solar no topo da atmosfera $\left(R_{a}\right)$ foi obtida da seguinte forma:

$$
\left.\left.\mathrm{R}_{\mathrm{a}}=37,6 \cdot(\overline{\mathrm{D}})^{2}\right)^{2}\left[\left(\mathrm{H} \cdot \frac{\pi}{180}\right) \operatorname{sen}(\phi) \cdot \operatorname{sen}(\delta)+\cos (\phi) \cdot \cos (\delta) \cdot \operatorname{sen} \mathrm{H}\right)\right]
$$

Em que: $\mathrm{R}_{\mathrm{a}}=$ radiação solar no topo da atmosfera $\left(\mathrm{MJ}^{-m^{-2}} \cdot \mathrm{dia}^{-1}\right) ;\left(\frac{\overline{\mathrm{D}}}{\mathrm{D}}\right)^{2}=$ distância relativa terra sol (U.A - Unidade Astrônomica); $\mathrm{H}=$ ângulo horário do por do sol $\left({ }^{\circ}\right) ; \phi=$ latitude $\left({ }^{\circ}\right) ; \delta=\operatorname{declinação~solar~}\left(^{\circ}\right)$.

Outras informações necessárias para processar o modelo são a latitude do local do plantio, fertilidade e textura do solo e capacidade mínima e máxima de retenção de água no solo. A variável referente à fertilidade do solo varia de 0 a 1 , sendo que 1 indica que há alta fertilidade e não impede o crescimento do plantio e se aproxima de 0 quando há redução da fertilidade. $\mathrm{Na}$ ocasião, foi considerado que não há impedimento da fertilidade do solo para desenvolvimento do plantio, atribuindo valor 1. A textura do solo foi obtida a partir do levantamento de solo realizado em campo, sendo constatada a predominância de latossolo vermelho com textura argilosa. Segundo Borges et al. (2012), nessa região há uma variação da disponibilidade de água no solo (Available Soil Water - ASW) de $200 \mathrm{~mm}$ a $260 \mathrm{~mm}$ na profundidade efetiva do sistema radicular de 2,0 metros.

\section{Validação da modelagem}

O 3-PG foi configurado para gerar estimativas mensais no período de junho de 2005 a dezembro de 2011, mesmo período de coleta dos dados de campo nas parcelas de inventário florestal. Para avaliar a acurácia das estimativas do modelo 3-PG foram utilizadas 335 medições do inventário florestal realizadas 
em 10 talhões e sobreposto aos valores médios estimados pelo 3-PG para avaliar o comportamento das variáveis diâmetro médio a altura do peito (DAP - $\mathrm{cm}$ ), altura $(\mathrm{m})$, volume $\left(\mathrm{m}^{3}\right)$ e incremento médio anual $\left(\right.$ IMA $\left.-\mathrm{m}^{3} \cdot \mathrm{ha}^{-1}\right)$. Posteriormente, as medições foram agrupadas nas diferentes idades do povoamento de forma que coincidissem com as idades de saída do modelo, totalizando 25 observações médias para aplicar os seguintes métodos estatísticos: correlação linear (Pearson) (r), coeficiente de determinação $\left(\mathrm{R}^{2}\right)$, erro padrão da estimativa $\left(\mathrm{S}_{\mathrm{yx}}\right)$ e erro padrão da estimativa em porcentagem $\left(\mathrm{S}_{\mathrm{yx}} \%\right)$, eficiência do modelo $(\mathrm{EF})$ e raiz quadrada do erro quadrático médio (RMSE) (SCOLFORO, 2005). As formulações seguem abaixo:

$$
\begin{aligned}
& \mathrm{r}=\frac{\sum \mathrm{O}_{\mathrm{i}} \mathrm{P}_{\mathrm{i}}}{\sqrt{\sum \mathrm{O}_{\mathrm{i}}^{2} \sqrt{\sum \mathrm{P}_{\mathrm{i}}^{2}}}} \\
& \mathrm{R}^{2}=1-\left(\frac{\mathrm{SQ}_{\mathrm{res}}}{\mathrm{SQ}_{\mathrm{t}}}\right) \\
& \mathrm{S}_{\mathrm{yx}}=\sqrt{\frac{\sum_{\mathrm{i}=1}^{\mathrm{n}}\left(\mathrm{P}_{\mathrm{i}}-\mathrm{O}_{\mathrm{i}}\right)^{2}}{\mathrm{n}-\mathrm{p}-1}} \quad \therefore \mathrm{S}_{\mathrm{yx}} \%=\frac{\mathrm{S}_{\mathrm{yx}}}{\overline{0}} * 100 \\
& \mathrm{EF}=1-\frac{\sum\left(\mathrm{O}_{\mathrm{i}}-\mathrm{P}_{\mathrm{i}}\right)^{2}}{\sum\left(\mathrm{O}_{\mathrm{i}}-\overline{0}\right)^{2}} \\
& \mathrm{RMSE}=\sqrt{\sum_{\mathrm{i}=1}^{\mathrm{n}} \frac{\left(\mathrm{P}_{\mathrm{i}}-\mathrm{O}_{\mathrm{i}}\right)^{2}}{\mathrm{n}}}
\end{aligned}
$$

Em que: $\mathrm{P}_{\mathrm{i}}=$ iésimo valor estimado pelo modelo 3-PG; $\mathrm{O}_{\mathrm{i}}=$ iésimo valor observado; $\bar{O}=$ média aritmética da variável observada; $S Q_{\text {res }}=$ soma dos quadrados dos resíduos; $S Q_{\mathrm{t}}=$ soma total dos quadrados; $\mathrm{n}=$ número de dados observados.

\section{RESULTADOS E DISCUSSÃO}

Os dados de saída do modelo 3-PG, com base na parametrização de Borges et al. (2012), foram plotados sobre os dados observados nos primeiros 7 anos do povoamento (Figura 1). Percebe-se que a distribuição dos dados estimados demonstra tendência de superestimativa em relação aos dados observados. Por ser um modelo pontual, os dados estimados devem ser próximos à média dos dados observados. Dessa forma, torna-se necessário ajustar e/ou parametrizar algumas variáveis para melhorar a acurácia do 3-PG.

O modelo tende a superestimar os valores das quatro variáveis avaliadas, principalmente após os 4 anos e meio de idade do povoamento (Figura 1). Após o quarto ano, espera-se que a altura média comece a estabilizar e o plantio aumente o incremento em diâmetro, isto porque o desenvolvimento do dossel se estabiliza e aumenta-se a competição entre plantas (LONDERO et al., 2015). Verifica-se que a modelagem dos dados por meio dos parâmetros propostos por Borges et al. (2012) tende a ter maiores resíduos próximo ao período de rotação do povoamento, o que fica evidente na relação entre os dados observados no inventário florestal e estimados pelo modelo 3-PG para o volume e IMA, cuja linha de tendência dos dados começa a se distanciar da linha 1:1 próximo aos 2 anos (Figura 1-f,h). 

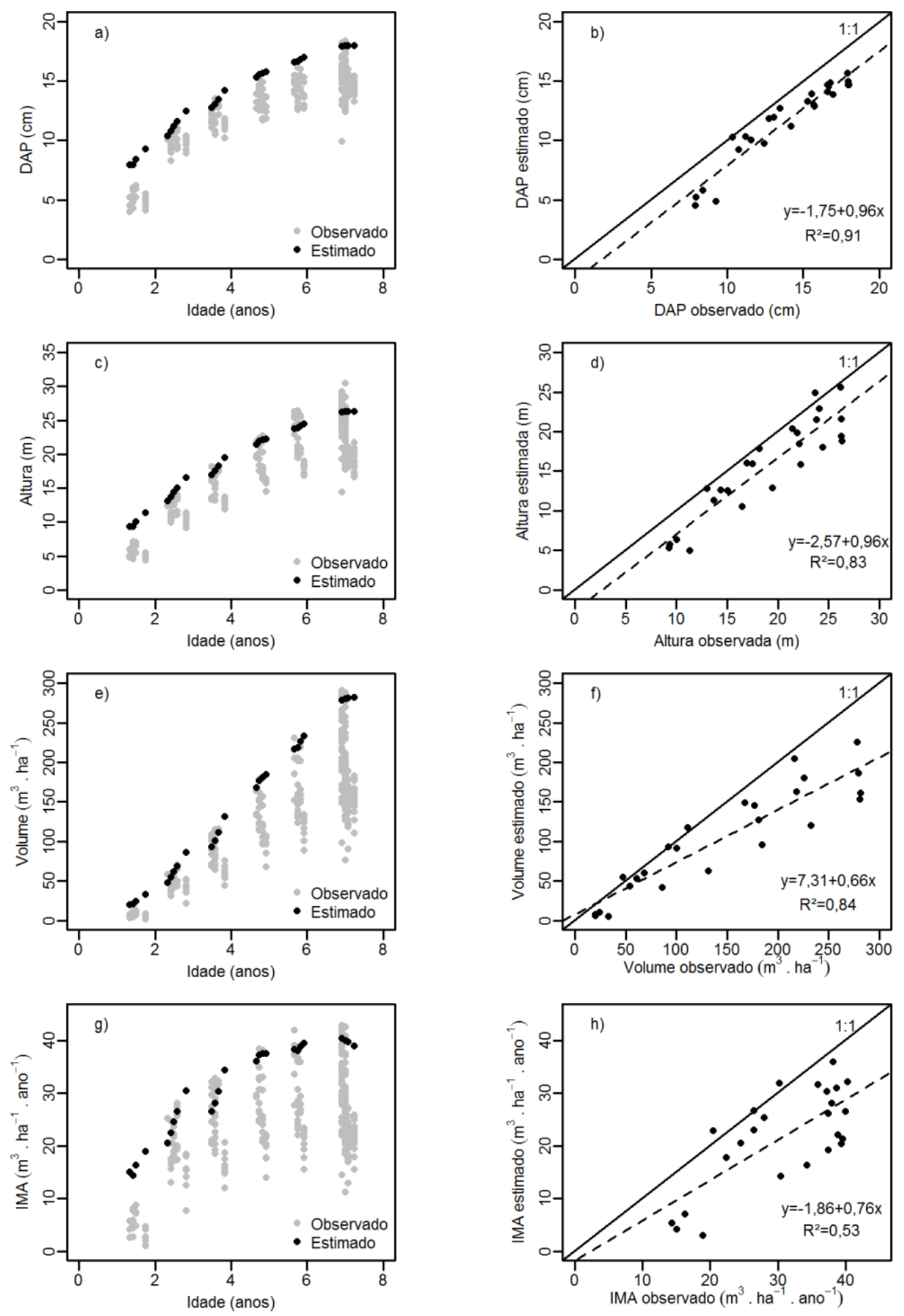

FIGURA 1: Distribuição das variáveis DAP (a), altura (c), volume (e) e IMA (g) em relação às idades do plantio de Eucalyptus spp. e a relação dos dados observados e estimados pelo modelo 3-PG para DAP (b), altura (d), volume (f) e IMA (h) para o município de Bocaiúva - MG, com a parametrização proposta por Borges et al. (2012).

FIGURE 1: Distribution of variables DBH (a), height (c), volume (e) and MAI (g) in relative ages of Eucalyptus spp. planting and relation of observed and estimated data by the 3-PG model to DBH (b), height (d), volume (f) and MAI (h) for the city of Bocaiúva (MG state) with the parameterization proposed by Borges et al. (2012). 
O coeficiente de correlação de Pearson foi relativamente alto para as variáveis DAP, altura e volume com valores superiores a 0,9 , enquanto para o IMA, esse coeficiente mostrou-se satisfatório $(r=0,73)$, demonstrando forte relação entre os dados observados e estimados pelo modelo (Tabela 1).

O coeficiente de determinação para as variáveis DAP, altura e volume foi superior a 0,8 , demonstrando que mais de $80 \%$ da variação da variável dependente é explicada pela variável independente (Figura 1). A eficiência do modelo mostrou-se muito baixa em todas as variáveis, não ultrapassando a marca de 0,5 para o DAP, altura e volume, enquanto que para o IMA o EF foi de - 0,53 , indicando que a média dos dados observados é melhor que a predição do modelo (Tabela 1).

TABELA 1: Resumo das análises estatísticas dos dados estimados para parametrização proposta por Borges et al. (2012).

TABLE 1: Summary of statistical analyses of the estimated data to parameterization proposed by Borges et al. (2012).

\begin{tabular}{lccccccc}
\hline \multicolumn{1}{c}{ Variáveis } & $\mathrm{N}$ & $\mathrm{r}$ & $\mathrm{R}^{2}$ & $\mathrm{~S}_{\mathrm{yx}}$ & $\mathrm{S}_{\mathrm{yx}} \%$ & $\mathrm{EF}$ & RMSE \\
\hline DAP $(\mathrm{cm})$ & 25 & 0,95 & 0,91 & 2,57 & 22,29 & 0,45 & 2,46 \\
Altura $(\mathrm{m})$ & 25 & 0,91 & 0,83 & 4,25 & 27,14 & 0,52 & 4,08 \\
Volume $\left(\mathrm{m}^{3} \cdot \mathrm{ha}^{-1}\right)$ & 25 & 0,92 & 0,84 & 59,47 & 58,18 & 0,22 & 57,04 \\
IMA $\left(\mathrm{m}^{3} \cdot\right.$ ha $^{-1} \cdot$.no $\left.^{-1}\right)$ & 25 & 0,73 & 0,53 & 11,66 & 53,78 & $-0,53$ & 11,18
\end{tabular}

Em que: N é o número de observações; $r$ é a correlação linear (Pearson); $\mathrm{R}^{2}$ é o coeficiente de determinação; $\mathrm{S}_{\mathrm{yx}}$ é o erro padrão da estimativa; $\mathrm{S}_{\mathrm{yx}} \%$ é o erro padrão da estimativa em percentual; $\mathrm{EF}$ é a eficiência do modelo; RMSE é a raiz do erro quadrático médio.

A fim de melhorar as estimativas do modelo, foram ajustados os modelos alométricos relativos às variáveis altura total $(\mathrm{H})$ e volume de madeira $(\mathrm{V})$ a partir de 2030 observações. Os ajustes dos modelos alométricos apresentaram resultados satisfatórios, sendo $\mathrm{R}^{2}$ de 0,92 e $\mathrm{S}_{\mathrm{yx}} \%$ de 10,48 para a variável altura e $\mathrm{R}^{2}$ de 0,87 e $\mathrm{S}_{\mathrm{yx}} \%$ de 21,59 para volume. Esses resultados são semelhantes aos encontrados por Londero et al. (2015), em Guaíba - RS, com Eucalyptus saligna.

Na Tabela 2 são apresentadas as equações, por meio das quais foram determinados os parâmetros constante de relacionamento entre altura e DAP $(\mathrm{aH})$, potência de relacionamento entre altura e DAP (nHB), constante de relacionamento entre volume e DAP $(\mathrm{aV})$, potência de relacionamento entre volume e DAP (nVB), respectivamente: $0,60,1,33,0,23,2,47$.

TABELA 2: Equações ajustadas para as variáveis altura $(\mathrm{H})$ e volume $(\mathrm{V})$ em função do diâmetro a altura do peito (DAP) para 2030 observações.

TABLE 2: Equations adjusted for the variables height $(\mathrm{H})$ and volume $(\mathrm{V})$ as a function of diameter at breast height (DBH) for 2030 observations.

\begin{tabular}{cccccc}
\hline Região & Variável & Equação & $\mathrm{N}$ & $\mathrm{R}^{2}$ & $\mathrm{~S}_{\mathrm{xy}} \%$ \\
\hline \multirow{2}{*}{ Bocaiúva $(\mathrm{MG})$} & altura $(\mathrm{m})$ & $\mathrm{H}=0,60^{*} \cdot$ dap $^{1,33^{*}}$ & 2030 & 0,92 & 10,48 \\
& volume $\left(\mathrm{m}^{3}\right)$ & $\mathrm{V}=0,23^{*} \cdot$ dap $^{2,47^{*}}$ & 2030 & 0,87 & 21,59
\end{tabular}

Em que: $\mathrm{N}=$ número de observações; $\mathrm{R}^{2}=$ coeficiente de determinação; $\mathrm{S}_{\mathrm{xy}} \%=$ erro padrão da estimativa em percentual; ${ }^{\text {ns }}=$ não significativo; $*$ = significativo a $5 \%$.

Segundo Rodríguez-Suárez, Iglesias e Diaz-Fierros (2010), as variáveis fullCanAge e StemPower $(\mathrm{nS})$, quando ajustadas adequadamente, proporcionam melhores resultados. A fullCanAge está relacionada com a idade de fechamento do dossel, ou seja, quando o dossel atinge $100 \%$ de cobertura. Os valores desses parâmetros podem ser obtidos através de medições em campo ou por meio de ajustes. Por meio de ajustes foi observado que aos 2 anos o dossel atinge o fechamento, ou seja, a partir dessa idade a RFA que chega ao dossel tem o máximo de interceptação pelo índice de área foliar (IAF). Versões anteriores do modelo 3-PG consideravam que toda RFA que chegava ao dossel era interceptado pelas folhas em qualquer idade. As versões mais recentes do modelo relacionam a fração de cobertura do solo pelo dossel (CanCover) desde a idade inicial até a idade em que o dossel tem máximo de cobertura (fullCanAge) (GONZALEZ-BENECKE et al., 2014). O valor do parâmetro fullCanAge, encontrado nesse estudo, corrobora os valores encontrados 
por Borges et al. (2012), enquanto que Rodríguez-Suárez et al. (2011) encontraram valor de 3,5 no noroeste (NW) da Espanha e Londero et al. (2015) encontraram 3 para o sul (S) do Brasil. Gonzalez-Benecke et al. (2014) mencionam que o parâmetro fullCanAge depende do material genético, do sítio de produção e do espaçamento. Como essa variável indica a idade de fechamento do dossel, percebe-se coerência nos resultados encontrados, já que as condições ambientais em região tropical proporcionam maior atividade fotossintética e, por conseguinte, maior incremento de fitomassa em menor intervalo de tempo, quando comparado às regiões temperadas. Rodríguez-Suárez, Iglesias e Diaz-Fierros (2010) constataram que as variáveis DAP e altura têm alta sensibilidade a variações do parâmetro fullCanAge, principalmente no período de 4 a 6 anos de idade, devido à característica assintótica do gênero Eucalyptus. Dessa forma, tornase necessária a adequação do valor desse parâmetro para povoamentos com rotações próximas aos 6 anos.

O parâmetro nS, que relaciona a biomassa do stem com o DAP, foi ajustado e obtido o valor de 3,22. Rodríguez-Suárez et al. (2011) mencionam que o parâmetro $\mathrm{nS}$ tem influência sobre as estimativas do modelo, principalmente após o quinto ano do plantio. Dessa forma, observa-se que na Figura 2 os dados estimados têm tendência central em relação aos dados observados, o que é esperado para um modelo que gera estimativas médias para as variáveis dendrométricas em diferentes idades do plantio. A Figura $2(\mathrm{~b}, \mathrm{~d}, \mathrm{e}, \mathrm{h})$ demonstra que a linha de tendência da regressão após o ajuste do modelo foi melhor, o que é demonstrando pela inclinação da curva próximo a 1. Almeida, Landsberg e Sands (2004) também observaram boa correspondência para as variáveis volume e área basal em plantio de Eucalyptus no Espírito Santo, Brasil.

Por meio da análise estatística, percebe-se melhoria nas estimativas, principalmente pela redução da variação dos dados, o que é demonstrado pelo erro padrão da estimativa $\left(\mathrm{S}_{\mathrm{yx}} \%\right)$ e a eficiência do modelo (EF) que para o DAP, altura e volume foram maiores que 0,80 (Tabela 3). Tickle et al. (2001) obtiveram $\mathrm{S}_{\mathrm{yx}} \%$ de $30 \%$, próximo ao encontrado neste trabalho para variável volume. Os valores do RMSE para as variáveis DAP e altura foram de 1,19 e 2,54, enquanto que Rodríguez-Suárez, Iglesias e Diaz-Fierros (2010) encontraram valores abaixo de 1,02 e 0,85 para as mesmas variáveis, porém, ajustando o modelo para três áreas localizadas em diferentes condições edáficas. Um dos fatores que pode ter contribuído para elevação do erro estatístico é a variação do tipo de solo entre talhões, no qual foram encontrados latossolo amarelo, latossolo vermelho, latossolo vermelho-amarelo e plintossolo, o que pode influenciar no balanço de água no solo e no desenvolvimento das raízes. 

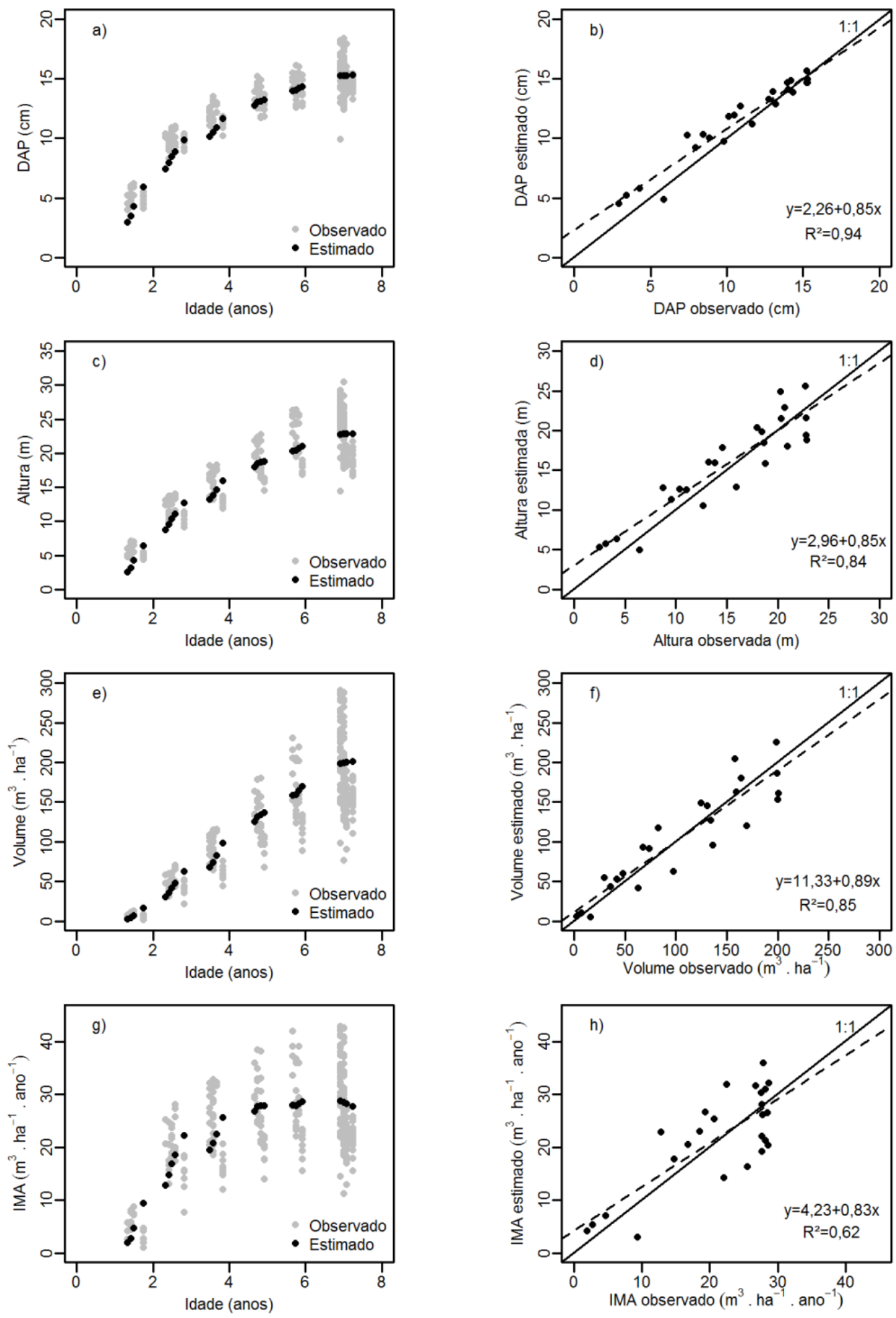

FIGURA 2: Distribuição das variáveis DAP (a), altura (c), volume (e) e IMA (g) em relação às idades do plantio e a relação dos dados observados e estimados pelo modelo 3-PG para DAP (b), altura (d), volume (f) e IMA (h) para o município de Bocaiúva - MG após o ajuste dos parâmetros fullCanAge, nS, aH, nHB, $\mathrm{aV}$ e $\mathrm{nVB}$.

FIGURE 2: Distribution of variables DBH (a), height (c), volume (e) and MAI (g) in relative ages of stand and relation of observed and estimated data by the 3-PG model to DBH (b), height (d), volume (f) and MAI (h) for the city of Bocaiúva (MG state) after adjusted of parameters fullCanAge, nS, aH, nHB, aV e nVB. 
O IMA apresentou melhoria considerável em relação à modelagem realizada com os parâmetros propostos na literatura para o Norte de Minas Gerais. Observa-se que o valor da EF do modelo ficou próximo a 0,60 e o RMSE foi de $5,82 \mathrm{~m}^{3} \cdot \mathrm{ha}^{-1} \cdot$ ano $^{-1}$ (Tabela 3 ). O coeficiente de determinação mostra que $62 \%$ da variação da variável dependente são explicadas pelas variáveis independentes, enquanto que Almeida et al. (2004) encontraram ajuste no qual a regressão explica mais de $80 \%$ dessa variação em uma análise com 10 observações. Os dados utilizados na validação das estimativas são provenientes de plantios com diferentes espécies e sítios de produção. Esses fatores podem reduzir a eficiência da regressão em explicar a variação dos dados devido aos fatores edafoclimáticos e genéticos, tornando-se necessário observar, também, o $\mathrm{S}_{\mathrm{yx}} \%$ e RMSE para avaliar o modelo. Segundo Stape, Ryan e Binkley (2004), o modelo 3-PG pode subestimar o incremento em período com baixa precipitação e superestimar nos anos com precipitação normal, devido à baixa sensibilidade do modelo ao abastecimento de água no solo e ao deficit de pressão de vapor (DPV), o que pode estar relacionado aos altos valores do $\mathrm{S}_{\mathrm{xy}} \%$ e RMSE encontrados para o IMA e volume (Tabela $3)$. Por ser um modelo gratuito, têm-se desenvolvido vários experimentos e propostas de modificações do 3-PG, a fim de melhorar sua acurácia, como o trabalho desenvolvido por Almeida e Sands (2015) em que propõem modificações no módulo relativo ao balanço de água no solo.

TABELA 3: Resumo das análises estatísticas dos dados estimados pelo modelo 3-PG após o ajuste dos parâmetros fullCanAge, $\mathrm{nS}, \mathrm{aH}, \mathrm{nHB}, \mathrm{aV}$ e nVB.

TABLE 3: Summary of statistical analyses of the data estimated by the model 3-PG after adjusting parameters fullCanAge, nS, aH, nHB, aV e nVB.

\begin{tabular}{cccccccc}
\hline Variáveis & $\mathrm{N}$ & $\mathrm{r}$ & $\mathrm{R}^{2}$ & $\mathrm{~S}_{\mathrm{yx}}$ & $\mathrm{S}_{\mathrm{yx}} \%$ & $\mathrm{EF}$ & $\mathrm{RMSE}$ \\
\hline DAP $(\mathrm{cm})$ & 25 & 0,97 & 0,94 & 1,24 & 10,74 & 0,87 & 1,19 \\
Altura $(\mathrm{m})$ & 25 & 0,92 & 0,84 & 2,76 & 17,59 & 0,80 & 2,64 \\
Volume $\left(\mathrm{m}^{3} \cdot \mathrm{ha}^{-1}\right)$ & 25 & 0,92 & 0,85 & 27,11 & 26,52 & 0,84 & 26,00 \\
IMA $\left(\mathrm{m}^{3} \cdot \mathrm{ha}^{-1} \cdot\right.$ ano $\left.^{-1}\right)$ & 25 & 0,78 & 0,62 & 6,07 & 28,00 & 0,58 & 5,82 \\
\hline
\end{tabular}

Em que: $\mathrm{N}=$ número de observações; $\mathrm{r}=$ correlação linear (Pearson); $\mathrm{R}^{2}=$ coeficiente de determinação; $\mathrm{S}_{\mathrm{yx}}$ = erro padrão da estimativa; $\mathrm{S}_{\mathrm{yx}} \%$ = erro padrão da estimativa em percentual; $\mathrm{EF}$ = eficiência do modelo; $\mathrm{RMSE}=$ raiz do erro quadrático médio; $\mathrm{DAP}=$ diâmetro da altura do peito; IMA = incremento médio anual.

O 3-PG tende a superestimar o DAP, H, volume e IMA médios nos dois primeiros anos (Figura 2-b,d,f,h). Após o segundo ano, os dados estimados se aproximam dos dados observados, com a linha de tendência dos dados estimados próximo à linha 1:1 e após os 6 anos o modelo tende a subestimar as variáveis dendrométricas. Esse comportamento também foi observado por Amichev, Johnston e Rees (2010) com híbridos de Walker aos 7 anos. Segundo Almeida et al. (2010), o 3-PG tende a superestimar essas variáveis quando se inicia o modelo e subestimar próximo aos 6 anos. Segundo esses autores, o 3-PG considera a densidade da madeira como variável de valor fixo para estimativa do volume e não considera a influência da precipitação ou estiagem sobre a densidade. Em estudo realizado no sul do Brasil em plantios de Eucalyptus saligna foi observado que em torno de 2 e 7 anos, os dados estimados foram próximos aos dados observados e no meio do ciclo os dados estimados foram um pouco superiores aos observados (LONDERO et al., 2015). Para Almeida e Sands (2015), a estimativa de produção em povoamento com diferentes clones pode reduzir a performance do modelo. A parametrização e ajuste do modelo com dados de uma espécie e em área com baixa variação de chuva pode minimizar os erros na estimativa das variáveis dendrométricas, devido à redução na variação da densidade da madeira. Nesse sentido, percebe-se que diferentemente dos modelos empíricos, o 3-PG pode captar os efeitos das variações climáticas sobre a produção, desde que sejam utilizados parâmetros com valores que expressem o potencial de crescimento da espécie e as características climáticas da região de interesse (Tabela 4). 
TABELA 4: Parâmetros do modelo 3-PG obtidos por ajuste e propostos por Borges et al. (2012) para Eucalyptus spp. no cerrado em Minas Gerais.

TABLE 4: $\quad$ Parameters of the model 3-PG obtained by fitting and proposed by Borges et al. (2012) for Eucalyptus spp. in the cerrado of Minas Gerais state.

\begin{tabular}{|c|c|c|c|c|}
\hline Parâmetro & Símbolo no 3-PG & Unidade & $\mathrm{F}$ & Dados \\
\hline Fração folha $/$ stem para DAP $=2 \mathrm{~cm}$ & pFS2 & - & $\mathrm{O}$ & 1,64 \\
\hline Fração folha $/$ stem para DAP $=20 \mathrm{~cm}$ & pFS20 & - & A & 0,15 \\
\hline Constante de relação entre a biomassa de stem e o DAP & StemConst & - & $\mathrm{O}$ & 0,02 \\
\hline Fração folha $/$ stem para DAP $=20 \mathrm{~cm}$ & StemPower & - & $\mathrm{O}$ & 3,22 \\
\hline Fração máxima da PPL para raízes & $\mathrm{pRx}$ & - & $\mathrm{L}$ & 0,5 \\
\hline Fração mínima da PPL para raízes & $\mathrm{pRn}$ & - & $\mathrm{L}$ & 0,1 \\
\hline Deposição de serapilheira para plantios em idade adulta & gammaFx & mês $^{-1}$ & A & 0,07 \\
\hline Deposição de serapilheira para $t=0$ & gammaF0 & mês ${ }^{-1}$ & $\mathrm{P}$ & 0,001 \\
\hline Idade em que a deposição de serapilheira = 1/2() & tgammaF & Mês & A & 4 \\
\hline Taxa média de ciclagem radicular & Rttover & mês ${ }^{-1}$ & A & 0,025 \\
\hline Temperatura mínima para o crescimento & Tmin & ${ }^{\circ} \mathrm{C}$ & $\mathrm{L}$ & 8 \\
\hline Temperatura ótima para o crescimento & Topt & ${ }^{\circ} \mathrm{C}$ & $\mathrm{L}$ & 25 \\
\hline Temperatura máxima para o crescimento & Tmax & ${ }^{\circ} \mathrm{C}$ & $\mathrm{L}$ & 40 \\
\hline Valor de $\mathrm{m}$ quando $\mathrm{FR}=0$ & $\mathrm{~m} 0$ & - & $\mathrm{P}$ & 0 \\
\hline Valor de quando $\mathrm{FR}=0$ & fNo & - & $\mathrm{L}$ & 0,5 \\
\hline Potência de (1 - FR), na expressão do & $\mathrm{fNn}$ & - & $\mathrm{L}$ & 1 \\
\hline Parâmetro relacionado com disponibilidade de água no solo & Soilclass & - & $\mathrm{O}$ & $\mathrm{C}$ \\
\hline Idade máxima do povoamento para computar a idade & MaxAge & ano & $\mathrm{L}$ & 9 \\
\hline Potência para idade relativa () & nAge & - & $\mathrm{P}$ & 4 \\
\hline Idade relativa igual a 0,5 & rAge & - & $P$ & 0,95 \\
\hline Área foliar específica para plantios em idade zero & SLA0 & $\mathrm{m}^{2} \cdot \mathrm{kg}^{-1}$ & $\mathrm{O}$ & 13,74 \\
\hline Área foliar específica para plantios em idade adulta & SLA1 & $\mathrm{m}^{2} \cdot \mathrm{kg}^{-1}$ & $\mathrm{O}$ & 7,56 \\
\hline Idade na qual a área foliar específica $=1 / 2($ SLA0 + SLA1 $)$ & tSLA & ano & $\mathrm{O}$ & 1,23 \\
\hline Coeficiente de extinção da RFAA pelo dossel & $\mathrm{K}$ & - & $\mathrm{P}$ & 0,5 \\
\hline Idade de fechamento do dossel & fullCanAge & ano & A & 2 \\
\hline Interceptação máxima de água pelo dossel & MaxIntcptn & - & $\mathrm{P}$ & 0,15 \\
\hline IAF em que a interceptação de água pelo dossel é máxima & LAImaxIntcptn & $\mathrm{m}^{2} \cdot \mathrm{m}^{-2}$ & $\mathrm{~L}$ & 0,15 \\
\hline Eficiência quântica máxima do dossel & Alpha & - & A & 0,08 \\
\hline Relação PPL/PPB & $\mathrm{Y}$ & - & $\mathrm{L}$ & 0,5 \\
\hline Condutância máxima do dossel & MaxCond & $\mathrm{m} \cdot \mathrm{s}^{-1}$ & $\mathrm{P}$ & 0,02 \\
\hline IAF em que a condutância do dossel é máxima & LAIgcx & $\mathrm{m}^{2} \cdot \mathrm{m}^{-2}$ & $\mathrm{P}$ & 3,33 \\
\hline Define a resposta estomática ao déficit de pressão do vapor & CoeffCond & $\mathrm{mBar}^{-1}$ & $\mathrm{~L}$ & 0,032 \\
\hline Condutância da camada limite do dossel & Blcond & $\mathrm{m} \cdot \mathrm{s}^{-1}$ & $\mathrm{P}$ & 0,2 \\
\hline Massa de stem máxima para cada 1000 árvores.ha $^{-1}$ & wSx 1000 & kg.arv ${ }^{-1}$ & $\mathrm{P}$ & 300 \\
\hline Fração de galho e casca para plantios em idade inicial & fracBB0 & - & $\mathrm{O}$ & 0,59 \\
\hline Fração de galho e casca para plantios em idade adulta & fracBB1 & - & $\mathrm{O}$ & 0,19 \\
\hline
\end{tabular}


TABELA 4: Continuação...

TABLE 4: Continued...

\begin{tabular}{lcccc}
\hline \multicolumn{1}{c}{ Parâmetro } & Símbolo no 3-PG & Unidade & $\mathrm{F}$ & Dados \\
\hline Idade na qual a fração de galhos e casca = 1/2(PPB0+PPB1) & tBB & ano & $\mathrm{O}$ & 2,17 \\
Densidade básica mínima - plantios em idade inicial & rhoMin & $\mathrm{t} . \mathrm{m}^{-3}$ & $\mathrm{O}$ & 0,382 \\
Densidade básica máxima - plantios em idade adulta & rhoMax & $\mathrm{t} \cdot \mathrm{m}^{-3}$ & $\mathrm{O}$ & 0,505 \\
Idade na qual a densidade básica = 1/2 () & tRho & ano & $\mathrm{O}$ & 2,264 \\
Constante de relacionamento entre altura e DAP & $\mathrm{aH}$ & - & $\mathrm{O}$ & 0,60 \\
Potência de relacionamento entre altura e DAP & $\mathrm{nHB}$ & - & $\mathrm{O}$ & 1,33 \\
Constante de relacionamento entre volume e DAP & $\mathrm{aV}$ & - & $\mathrm{O}$ & 0,23 \\
Potência de relacionamento entre volume e DAP & $\mathrm{nVB}$ & - & $\mathrm{O}$ & 2,47 \\
\hline
\end{tabular}

Em que: $\mathrm{F}$ = forma de obtenção do parâmetro; $\mathrm{A}=$ parâmetro ajustado; $\mathrm{O}=$ observado; $\mathrm{L}=$ parâmetro obtido da literatura; $\mathrm{P}=$ parâmetro com valor original do modelo; $\mathrm{DAP}=$ diâmetro a altura do peito; $\mathrm{PPL}=$ Produtividade Primária Líquida; $\mathrm{FR}=$ razão de fertilidade; $\mathrm{RFAA}=$ radiação fotossinteticamente ativa absorvida; $\mathrm{PPB}=$ produtividade primária bruta;

A Tabela 4 apresenta os valores dos parâmetros do 3-PG necessários para realizar estimativas do potencial produtivo de novas áreas com plantios de eucalipto, podendo-se, também, criar cenários de produção florestal frente a variações nas práticas de manejo como adubação ou desbaste, avaliar os impactos de mudanças climáticas ou a variabilidade climática sobre a produção florestal (ALMEIDA et al., 2004), devendo-se inserir dados relativos a variáveis climatológicos que caracterizam a região.

\section{CONCLUSÕES}

O modelo 3-PG foi eficiente na estimativa de variáveis de interesse no manejo florestal, neste estudo. Utilizando-se parâmetros obtidos da literatura, dados provenientes de EMA e sensoriamento remoto mostra que os resultados podem caracterizar o potencial produtivo da região, sendo necessário o ajuste de alguns parâmetros para melhorar as estimativas do modelo.

Com base em dados de inventários florestais podem-se parametrizar os modelos alométricos relativos à altura e volume, de forma que juntamente com o ajuste dos parâmetros fullCanAge e StemPower obtiveram-se estimativas satisfatórias para as variáveis diâmetro a altura do peito (DAP), altura e volume. O incremento médio anual (IMA) apresentou variação considerável, que pode estar relacionado a variações edafoclimáticas nos diferentes talhões de produção.

Assim, o modelo 3-PG demonstra que pode ser utilizado como ferramenta do planejamento florestal, auxiliando a tomada de decisão sobre a definição e mapeamento de áreas com potencial para a produção florestal.

\section{REFERÊNCIAS}

ALLEN, R. G.; JENSEN, M. E.; BORNAN, R. D. Operational estimates of reference evapotranspiration. Agronomy Journal, Madison, v. 81, n. 4, p. 650-662, 1989.

ALMEIDA, A. C. et al. Mapping the effect of spatial and temporal variation in climate and soils on Eucalyptus plantation production with 3-PG, a process-based growth model. Forest Ecology and Management, Amsterdam, v. 259, n. 9, p. 1730-1740, 2010.

ALMEIDA, A. C. et al. Needs and opportunities for using a process-based productivity model as a practical tool in Eucalyptus plantations. Forest Ecology and Management, Amsterdam, v. 193, n. 1-2, p. 167-177, 2004.

ALMEIDA, A. C.; LANDSBERG, J. J.; SANDS, P. J. Parameterisation of 3-PG model fast-growing Eucalyptus grandis plantations. Forest Ecology and Management, Amsterdam, v. 193, n. 1-2, p. 179-195, 2004. 
ALMEIDA, C. A.; SANDS, P. J. Improving the ability of 3-PG to model the water balance of forest plantations in contrasting environments. Ecohydrology, v. 9, n. 4, p. 610-630, 2015.

AMICHEV, B. Y.; JOHNSTON, M.; REES, K. C. J. V. Hybrid poplar growth in bioenergy production systems: Biomass prediction with a simple process-based model (3PG). Biomass \& Bioenergy, Oxford, v. 34, n. 5, p. 687-702, 2010.

BORGES, J. S. et al. Parameterization of the 3-PG model for eucalypt in the region of cerrado in Minas Gerais state. Ciência Florestal, Santa Maria, v. 22, n. 3, p. 567-578, 2012.

CAMPION, J. M.; ESPREY, L. J.; SCHOLES, M. C. Application of the 3-PG model to a Eucalyptus grandis stand subjected to varying levels of water and nutritional constraints in KwaZulu-Natal, South Africa. The Southern African Forestry Journal, África do Sul, v. 203, n. 1, p, 3-13, 2005.

COOPS, N. C. et al. Comparison of MODIS, eddy covariance determined and physiologically modelled gross primary production (GPP) in a Douglas-fir forest stand. Remote Sensing of Environment, New York, v. 107, n. 3, p. 385-401, 2007.

COOPS, N. C.; WARING, R. H.; HILKER, T. Prediction of soil properties using a process-based forest growth model to match satellite-derived estimates of leaf area index. Remote Sensing of Environment, New York, v. 126, n. 1, p. 160-173, 2012.

GONZALEZ-BENECKE, C. A. et al. Parameterization of the 3-PG model for Pinus elliottii stands using alternative methods to estimate fertility rating, biomass partitioning and canopy closure. Forest Ecology and Management, Amsterdam, v. 327, n. 1, p. 55-75, 2014.

LANDSBERG, J. J.; WARING, R. H. A generalized model of forest productivity using simplified concepts of radiation-use efficiency, carbon balance and partitioning. Forest Ecology and Management, Amsterdam, v. 95 , n. 3, p. 209-228, 1997.

LONDERO, E. K. et al. Calibração do modelo 3-PG para Eucalyptus saligna Smith na região de Guaíba-RS. Ciência Florestal, Santa Maria, v. 25, n. 2, p. 293-305, 2015.

MAESTRI, R. Modelo de crescimento e produção para povoamentos clonais de Eucalyptus grandis considerando variáveis ambientais. 2003. 143 f. Tese (Doutorado em Ciências Florestais) - Universidade Federal do Paraná, Curitiba, 2003.

MÄKELÄ, A. et al. Process-based models for forest ecosystem management: current state of the art and challenges for practical implementation. Tree Physiology, Oxford, v. 20, n. 5-6, p. 289-298, 2000.

MCMURTRIE, R. E.; ROOK, D. A.; KELLIHER, F. M. Modelling the yield of Pinus radiate on a site limited by water and nitrogen. Forest Ecology and Management, Amsterdam, v. 30, n. 1-4, p. 381-413, 1990.

NIGHTINGALE, J. M. et al. Use of 3-PG and 3-PGS to simulate forest growth dynamics of Australian tropical rainforests I. Parameterisation and calibration for old-growth, regenerating and plantation forests. Forest Ecology and Management, Amsterdam, v. 254, n. 2, p. 107-121, 2008.

NOLÈ, A. et al. Assessing temporal variation of primary and ecosystem production in two Mediterranean forests using a modified 3-PG model. Annals of Forest Science, Paris, v. 70, n. 7, p. 729-741, 2013.

OLIVEIRA JÚNIOR, J. F. et al. Análise da Precipitação e sua Relação com Sistemas Meteorológicos em Seropédica, Rio de Janeiro. Floresta e Ambiente, Seropédica, v. 21, n. 2, p. 140-149, 2014.

PAUL, K. I. et al. Calibration of the forest growth model 3-PG to eucalypt plantations growing in low rainfall regions of Australia. Forest Ecology and Management, Amsterdam, v. 243, n. 2-3, p. 237-247, 2007.

RODRÍGUEZ-SUÁREZ, J. A.; IGLESIAS, M. L.; DIAZ-FIERROS, F. Application of the 3PG forest growth model to a Eucalyptus globulus plantation in Northwest Spain. European Journal of Forest Research, Heidelberg, v. 129, n. 4, p. 573-583, 2010.

RUNNING, S. W.; COUGHLAN, J. C. A general model of forest ecosystem processes for regional applications I. Hydrologic balance, canopy gas exchange and primary production processes. Ecological Modelling, Amsterdam, v. 42, n. 2, p. 125-154, 1988.

SANDS, P. J.; LANDSBERG, J. J. Parameterisation of 3-PG for plantation grown Eucalyptus globulus. Forest Ecology and Management, Amsterdam, v. 163, n. 2-3, p. 273-292, 2002.

SCOLFORO, J. R. S. Biometria florestal: Parte I: modelos de regressão linear e não-linear; Parte II: modelos para relação hipsométrica, volume, afilamento e peso de matéria seca. Lavras: UFLA/FAEPE, 
2005. $352 \mathrm{p}$.

STAPE, J. L. et al. The Brazil Eucalyptus Potential Productivity Project: Influence of water, nutrients and stand uniformity on wood production. Forest Ecology and Management, Amsterdam, v. 259, n. 9, p. 1684-1694, 2010.

STAPE, J. L.; RYAN, M. G.; BINKLEY, D. Testing the utility of 3-PG model for growth of Eucalyptus grandis $\mathrm{x}$ urophylla with natural and manipulated supplies of water and nutrients. Forest Ecology and Management, Amsterdam, v. 193, n. 1-2, p. 219-234, 2004.

TICKLE, P. K. et al. Assessing forest productivity at local scales across a native eucaluypt forest using a process model, 3PG-SPATIAL. Forest Ecology and Management, Amsterdam, v. 152, n. 1-3, p. 275-291, 2001.

WEI, L. et al. 3-PG simulations of young ponderosa pine plantations under varied management intensity: Why do they grow so differently? Forest Ecology and Management, Amsterdam, v. 313, n. 1, p. 69-82, 2014.

WEINSTEIN, D. A.; BELOIN, R. M.; YANAI, R. D. Modeling changes in red spruce carbon balance and allocation in response to interacting ozone and nutrient stresses. Tree Physiology, Oxford, v. 9, n. 1-2, p. 127-146, 1991. 